\title{
Polycystic Ovarian Syndrome and Female Infertility
}

\author{
Syed Manzar Abbas Shah Naqvi1 ${ }^{*}$, Januka Bhusal Bhattarai2*, Hui Li², Xian Wang Wang1,2\# \\ ${ }^{1}$ The Second School of Clinical Medicine, Health Science Center, Yangtze University, Jingzhou, China \\ ${ }^{2}$ Department of Obstetrics and Gynecology, The Second Affiliated Hospital of Yangtze University \& Jingzhou Central Hospital, \\ Jingzhou, China \\ Email: syedmanzar604@gmail.com, j_bhusal@yahoo.com, 70169683@qq.com, ”xwshine@yangtzeu.edu.cn
}

How to cite this paper: Naqvi, S.M.A.S., Bhattarai, J.B., Li, H. and Wang, X.W. (2020) Polycystic Ovarian Syndrome and Female Infertility. Yangtze Medicine, 4, 11-27.

https://doi.org/10.4236/ym.2020.41002

Received: May 6, 2019

Accepted: September 14, 2019

Published: September 17, 2019

Copyright $\odot 2020$ by author(s) and Scientific Research Publishing Inc. This work is licensed under the Creative Commons Attribution International License (CC BY 4.0).

http://creativecommons.org/licenses/by/4.0/ (c) (i) Open Access

\begin{abstract}
Polycystic ovarian syndrome (PCOS) is the most common endocrine disorder in women of reproductive age, associated with chronic anovulation and hyperandrogenism. The cause of PCOS is multi-factorial and the mechanism is still unclear. A number of complications are closely related with PCOS such as infertility, hairy and obesity. Approximately up to one-third of females with PCOs have issues with infertility. Obesity is an independent factor which exacerbates infertility in PCOS, reduces the efficacy of infertility treatment and provokes a greater risk of miscarriage. Preconception counseling and guidelines play an initial major role in infertility treatment. The option of the most appropriate treatment depends upon various factors such as age, tubal patency, quality of sperm, experience, and duration of previous treatments and the level of anxiety of the couple. Tubal patency test and semen analysis are mandatory to decide the suitable treatment. Treatment modalities have two measures-non-pharmacological and the pharmacological. Oral clomiphene citrate remains the first line treatment to trigger ovulation. Gonadotrophin and laparoscopic ovarian drilling (LOD) surgery are the other treatment option. In vitro fertilization (IVF) and embryo transfer are the third line treatment and IVF remains the first option in the patient with diagnosed and established bilateral tubal occlusion and/or concentration of recovered motile sperm less than or equal to 5 million. Here, etiology, pathophysiology and molecular mechanisms of PCOS are reviewed, and perspectives are given for further research. The recent development, treatment, and diagnosis of PCOS are also highlighted. The summary will be of guiding significance in the prevention and treatment of PCOS.
\end{abstract}

\section{Keywords}

Polycystic Ovary Syndrome (PCOS), Anovulation, Clomiphene Citrate, Infertility

\footnotetext{
${ }^{\star}$ Authors contributed equally.

${ }^{*}$ Corresponding authors.
} 


\section{Introduction}

The polycystic ovary syndrome (PCOS) is the most frequent multisystem endocrinopathy among women of reproductive age. It was discovered and named for the first time by Stein and Leventhal in 1935 [1] [2] [3]. In the publication, Stein and Leventhal identified the phenotype of seven cases of PCOS in women with oligo/amenorrhea, hirsutism, obesity, and polycystic ovaries bilaterally [1] [4] [5]. The current incidence of PCOS (5\% - 6\%) is getting increasingly fast due to stress and change in lifestyle, also becoming a common problem among adolescent girls developing soon after the puberty [5] [6]. Infertility is considered as the devastating issue for the women of reproductive age group and it may be due to numerous reasons [4].

PCOS is frequently associated with reproductive complications importantly infertility which accounts for around $6 \%-21 \%$ [7]. Most of the women may accidentally know and realize that they have PCOS only when they seek infertility therapy [7]. Some latest research has shown diminished sensitivity of the gonadotropin-releasing hormone ( $\mathrm{GnRH})$ pulse generator to inhibition by ovarian steroids, mostly progesterone [8] [9]. Women with polycystic ovary syndrome (PCOS) need higher levels of progesterone to slow the frequency of GnRH pulse secretion, resulting in insufficient plasma follicle-stimulating hormone (FSH) synthesis and persistent plasma luteinizing hormone (LH) stimulation of ovarian androgens. In hyperandrogenemic girls certain to develop PCOS, increase in ovarian steroids may not be adequate to suppress the GnRH pulse generator, leading to a persistently rapid LH pulse frequency, reduced FSH generation, and insufficient follicular development [8] [9]. These imbalances are the reason behind oligomenorrhea or amenorrhea (infrequent or lack of menstruation) [10]. There is a number of additional clinical features, like tiny cysts on the surface of the ovaries (polycystic) and hair symptoms i.e.: hirsutism due to underproduction of estrogen and overproduction of androgens (testosterone, dehydroepiandrosterone and androstenedione) by the ovaries and skin symptoms i.e.: acanthosis nigra due to insulin resistance and hyperinsulinemia [4] [11] [12] [13]. If a woman conceives, carbohydrate intolerance, hypertension may develop during pregnancy; $20 \%-30 \%$ of pregnancy in PCOS females are ending up with first-trimester spontaneous abortion [13] [14] [15].

\section{Etiology of PCOS}

Although PCOS has been attributed to several factors including environmental factors such as an unhealthy diet and lack of physical activity, change in lifestyle and stress, the exact cause of PCOS is still unknown, but it is recognized to be a multi-factorial condition with a genetic and familial component (Figure 1) [13] [16] [17] [18]. Approximately $20 \%$ - $40 \%$ of primary female relatives of women with PCOS go on to develop PCOS themselves means familial occurrence with a reported-linked dominant mode of inheritance is also involved [19] [20]. Many women with PCOS have female relatives with PCOS, even if it was never diagnosed 


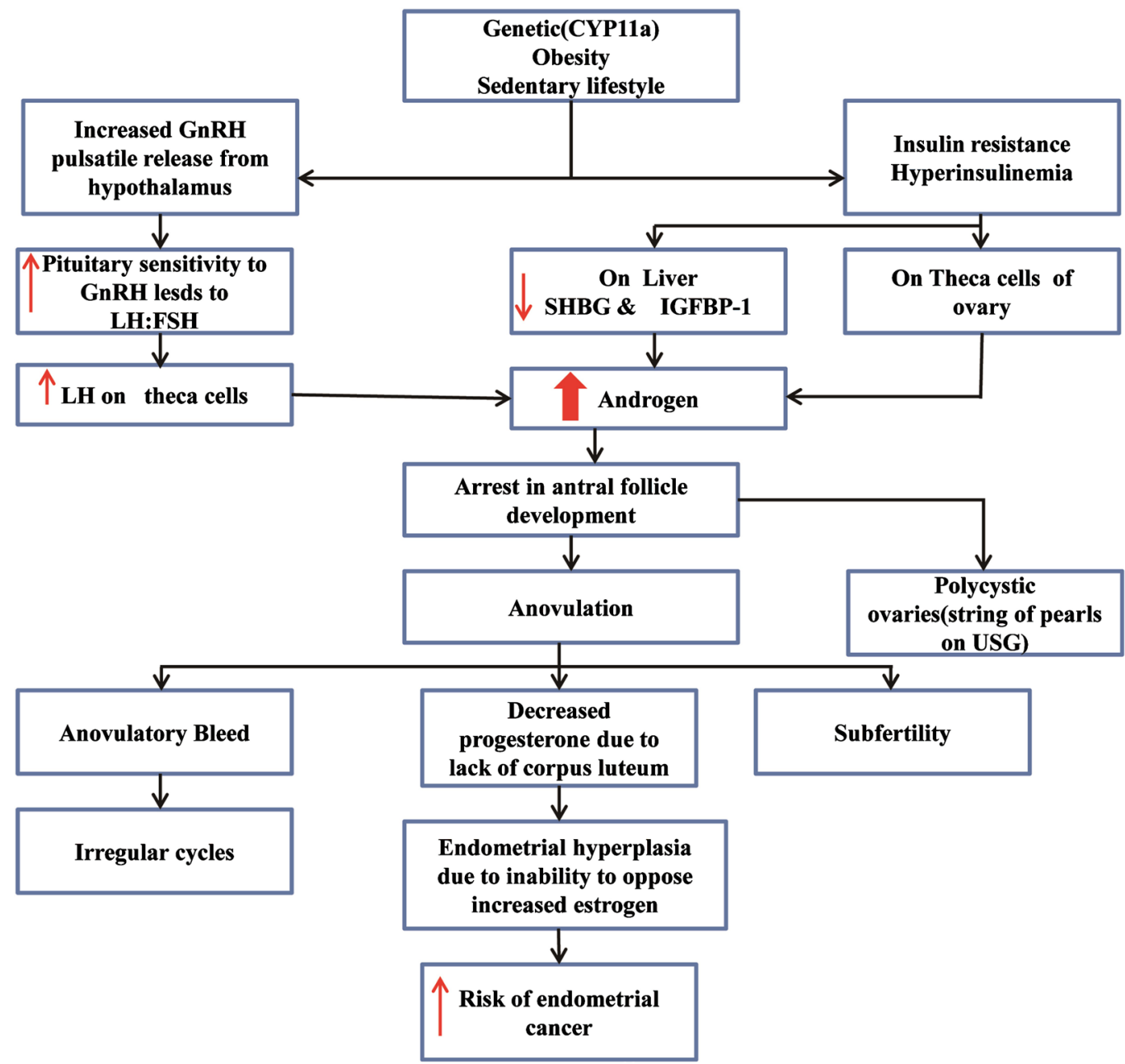

Figure 1. Pathophysiology up to long term complications of PCOS. GnRH = Gonadotropin releasing hormone; $\mathrm{LH}=$ Luteinizing hormone; FSH = Follicular stimulating hormone; SHBG = Sex hormone binding globulin; IGBFP = Insulin like growth factor binding protein; $\uparrow=$ increase; $\downarrow=$ decrease; $=$ increase more.

[21] [22]. As with type II diabetes, it is likely that numerous genes each make a small contribution to the etiology of PCOS; and recent genome-wide association studies have identified candidate genes [4] [13] [21] [23]. Any underlying genetic predisposition is likely complicated by epigenetic and the gene (CYP11a) mutation has been discovered in this connection [9] [24] [25]. High maternal serum testosterone concentrations during pregnancy may influence on the behavior of children like autism disorders and raised level anti-Mullerian hormone (AMH) in adolescence. They are also supposed to develop the female reproductive axis to induce the features of PCOS in later life: oligo/anovulation, polycystic ovaries, hyperandrogenism and insulin resistance (IR) [26]

\section{PCOS Pathophysiology Regarding Infertility}

\subsection{Normal Oogenesis and Ovulation}

Development of the ovaries begins in intrauterine life, as early as the $3^{\text {rd }}$ weeks of 
gestation, primordial germ cells appear in the endoderm of yolk sac migrate along the dorsal mesentery to the urogenital ridge by amoeboid movement. As shown in Figure 2, by the $8^{\text {th }}$ weeks, the primitive germ cells undergo multiple rapid mitotic divisions and get differentiated into oogonia, number of oogonia reaches its maximum at $20^{\text {th }}$ weeks. The mitotic division gradually ceases and the majority enters into the prophase of first meiotic division are called primary oocytes. These primary oocytes are surrounded by flat cells (granulosa cells) and are called primordial follicles. At birth these oogonia get replaced by primary oocytes; hence there are no more mitotic divisions. Thus all those primordial follicles with primary oocytes in the ovary of newborn female are getting arrested in the diplotene stage prophase of first meiotic division till puberty. At puberty, now the arrested primary oocyte with surrounding follicular cells (also known as primordial follicle) gets developed into mature Graafian follicle (measures its size about $12-16 \mathrm{~mm}$ ) through the development of growing follicle, primary follicle(with multilayered follicular cells) and secondary follicle (primary follicle with antrum) under the control of FSH secreted by anterior pituitary gland. The

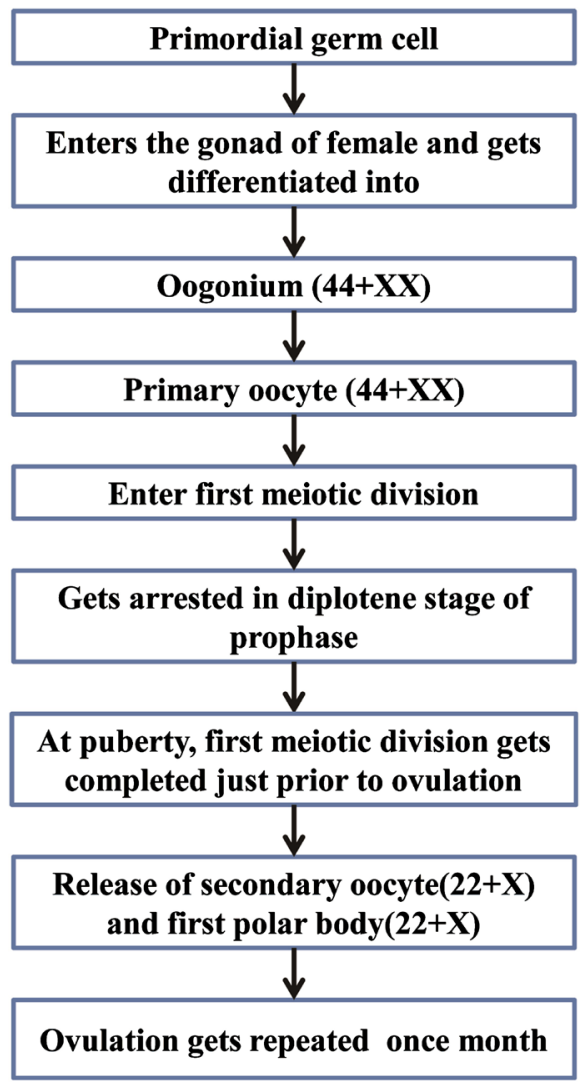

Figure 2. Normal ovulation process. The primitive germ cells undergo multiple rapid mitotic divisions and get differentiated into oogonia. The mitotic division gradually ceases and the majority primitive germ cells enter into the prophase of first meiotic division are called primary oocytes. These primary oocytes shift to the first meiotic division and get arrested in diplotene stage of prophase, the result of first meiotic division release secondary oocyte $(22+\mathrm{X})$ and first polar body $(22+\mathrm{X})$, and ovulation gets repeated once monthly. 
mature Graafian follicle is spherical or ovoid in shape contains liquor folliculi. The lining of it consists of two layers, the theca cells layer and granulose cell layer [10]. Theca is responsible for the production of ovarian hormones; ie; estrogen, progesterone and sometimes extended to the production of androgens. Granulosa cells secrete a clear fluid composed of protein, known as liquor folliculi, which contains ovarian hormone estrogen. Until puberty, most primordial follicles in the ovary undergo retrogression by a process termed as follicle atresia. During reproductive age, several follicles tend to develop in each menstrual cycle. In response to FSH, small gap junctions (to provide a pathway for nutrition and metabolic interchange) develop between the granulosa cells and the oocyte. Among several follicles developing on both ovaries, one follicle grows faster than the rest and generates more FSH receptors and estrogen. The raised level of estrogen stimulates LH receptors in the theca cells as well as gives negative feedback to the anterior pituitary gland leading to progressive fall in FSH level, due to which only developed follicle remains and undergoes ovulation, rest all follicles get atrophied [27] [28].

\subsection{Oogenesis in PCOS and Infertility}

The oogenesis process is completely different than that of a normal cycling fertile woman. During folliculogenesis, the individual conversion of the primordial follicle to primary follicle does not depend upon the gonadotropins [13] [29] [30]. Due to the functional deficiency of the endogenous action of FSH, the follicular growth is frustrated at different phases of maturation (2-10 $\mathrm{mm}$ in diameter) [5]. In the obese patient, the fat (adipose tissue) secretes leptin (also secreted by ovarian follicle), adiponectin and cytokines which interferes the insulin signaling pathways in liver and muscles which results in insulin resistance and hyperinsulinemia. Insulin stimulates the LH to cause thecal hyperplasia and secretes androgens, testosterone, and epi-androstenedione. Due to the hyperactivity of thecal cell and increase level of androgens, testosterones, and epi-androstenedione, accelerate the generation of estrogen in granulosa cells [31] [32] [33]. There is defective FSH induced aromatization of androgens to estrogens; therefore follicular microenvironment is more androgenic rather than estrogenic. Androgen also suppresses the growth of the dominant follicle and prevents apoptosis of smaller follicles which are normally destined to disappear in the late follicular phase; hence multiple immature follicles remain in the ovary. Unless there is estrogenic follicular microenvironment, follicular growth, maturation, and ovulation cannot occur. Epi-androstenedione converts to estrone in the peripheral fat, leads to a rise in estrogen and inhibin level, in turn, causing tonically elevated LH without any surge. LH surge is essential for ovulation to occur. In PCOs patient, a spike increase of LH can also cause early maturation of granulosa cells rather than the organized signaling to continue follicular development from the primary to the secondary than to graffian follicular stage [34]. Estrone level is prompted whereas estradiol remains normal, resulting in the raised ratio of es- 
trone/estradiol.

Hyperandrogenism due to insulin resistance and hyperinsulinemia in PCOs patient occurs due to following two ways- by stimulating the synthesis of androgen from ovary along with LH [17] or by decreasing the sex hormone binding globulin (SHBG) [35]. So hyperandrogenism again lowers the level of hepatic sex hormone binding globulin, hence level of free testosterone gets raised. Clinically hyperandrogenism is primarily manifested by hirsutism, acne and male pattern alopecia [36]. Hirsutism is the male type terminal hair growth and distribution in females [37]. PCOS is a common cause of hirsutism occurring in approximately $60 \%$ of cases depending upon the race and degree of obesity [28] [36]. Hirsutism should be assessed with a standardized scoring system (Ferriman-Gallwey score) [38].

The pathogenesis is initiated from the early adolescent period; early adrenarche and early menarche are observed in a few girls. Menstrual abnormalities like oligomenorrhoea or secondary amenorrhea more than 3 months followed by menometrorrhagia are the clinical manifestation of ovarian dysfunction ie: anovulation or oligo ovulation [39]. PCOS is the most common cause of an ovulatory infertility. About $90 \%$ to $95 \%$ of women with anovulation who attends infertility clinics are diagnosed to be PCOS. But $60 \%$ of women with PCOS are found to be able to conceive within 12 months, although time to conceive is often increased [9]. In the fact that all women with PCOS are usually not entirely sterile but they do ovulate sometimes spontaneously. The ovulatory frequency has not been studied well, but few studies show that ovulation occurs in up to $32 \%$ of menstrual cycles [11]. In those with PCOS and infertility, $90 \%$ are overweight. Obesity is another independent factor which exacerbates infertility, reduces the efficacy of infertility treatment and induces a greater risk of miscarriage [39]. Currently, an active debate about the appropriate limit for body mass index for assisted reproduction therapies is going on, which gives a clear idea that in overweight women, the pregnancy success rate is reduced along with the association of risk in pregnancy [40]. Ideally, Overweight has to be optimized before conception. Age is also a factor which exacerbates infertility, so it is wise to think about family planning on time.

The diagnosis of PCOS is based on the Rotterdam criteria (Table 1). In Rotterdam,

Table 1. Rotterdam criteria for PCOS [41] [42].

\begin{tabular}{|c|c|}
\hline \multirow{5}{*}{$\begin{array}{l}\text { 2. Clinical and/or biochemical } \\
\text { signs of hyperandrogenism }\end{array}$} & Biochemical \\
\hline & Total testosterone $>70 \mathrm{ng} / \mathrm{dl}$ \\
\hline & Androstenedione $>245 \mathrm{ng} / \mathrm{dl}$ \\
\hline & DHEA-S > 248 ng/dl \\
\hline & Clinical: Acne, Hirsutism, Acanthosis nigra \\
\hline
\end{tabular}


ESHRE (European Society of Human Reproduction and Embryology)/ASRM (American Society of Reproductive Medicine), the PCOS consensus workshop group has proposed a revision of the PCOS diagnostic criteria which suggests the presence of at least two of the following criteria together with the exclusion of other etiologies (congenital adrenal hyperplasia, hyperprolactinemia, thyroid dysfunction, androgen-secreting tumors and Cushing syndrome) [21].

There is a remarkable difference between normal and polycystic ovary (Figure 3). Transvaginal ultrasound is the most widely used technique for the ultrasound assessment of PCOS. The comparison of ultrasound imaging between the normal ovary and polycystic ovary is shown in figure (Figure 4). The sonographic criteria have been subsequently modified and, therefore, the increase in ovarian volume $\left(>10 \mathrm{~cm}^{3}\right)$ with dense echogenic stroma due to enlargement and the

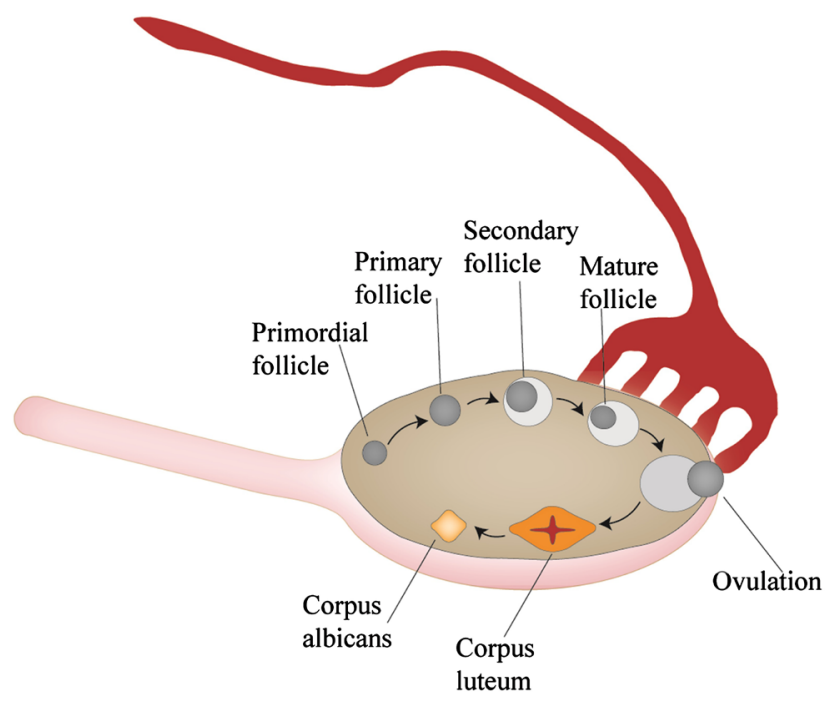

Normal Ovary

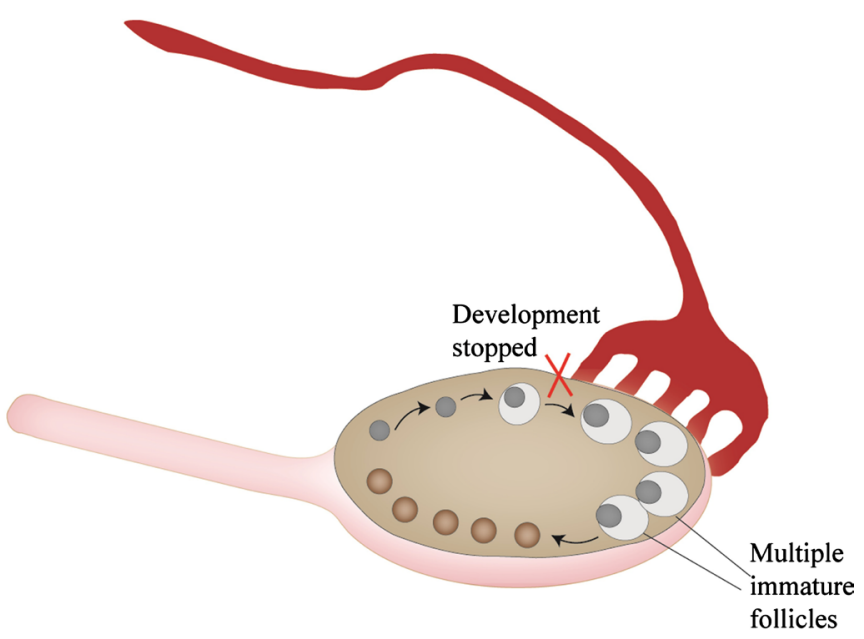

Polycystic 0vary

Figure 3. Gross anatomical comparison between normal ovary showing normal oogenesis with Polycystic ovary showing arrested follicular development and lack of ovulation (source of figure https://www.indeedcare.com/blog/dont-let-pcos-interfere-with-your-life/).
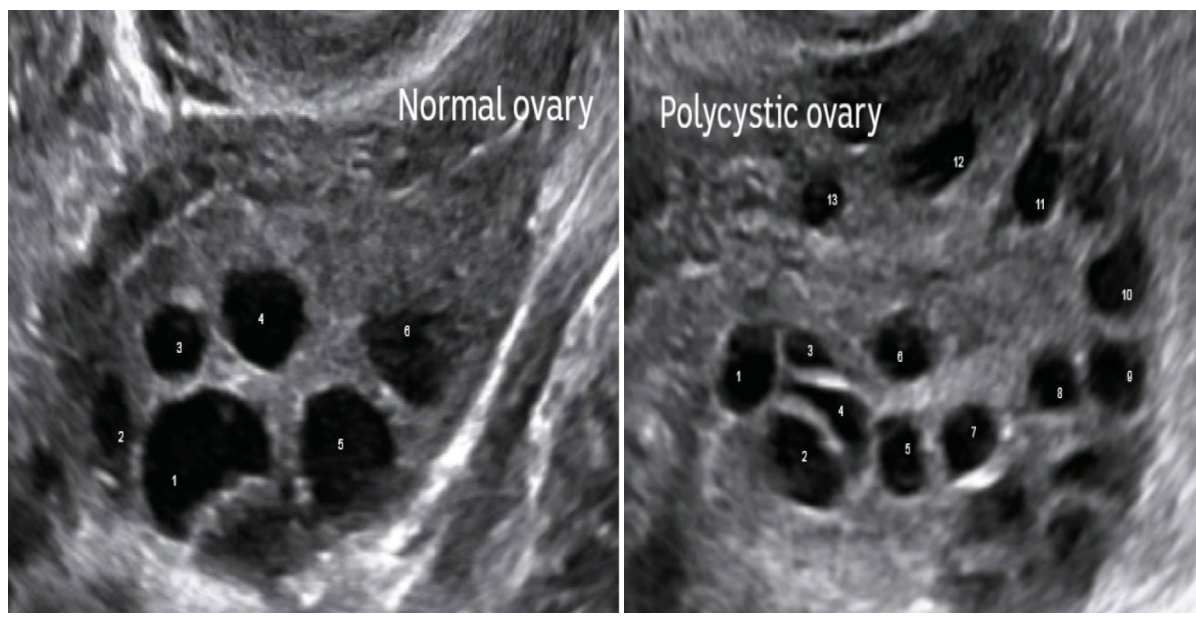

Figure 4. Comparison of ultrasound imaging between the normal ovary and polycystic ovary (necklace appearance), source of Figure 4, it is taken from Jingzhou central Hospital. 
presence of multiple (12 or more) follicles each with a diameter of 2 to $9 \mathrm{~mm}$ sized placed peripherally along the surface of ovary giving a necklace appearance at least in one ovary [43]. It must be excluded from this rule women who use an oral contraceptive as it transforms the ovarian morphology in healthy women and probably also in women with PCO [21]. The ultrasound findings can rule out the ovarian tumor, adrenal hyperplasia or tumor and also shows endometrial hyperplasia if present. The ultrasound should preferably be performed in the early follicular phase. Laparoscopy is reserved for therapeutic purpose rather that diagnostic purpose because the diagnosis can be confirmed by ultrasound findings.

\section{Clinical Pictures of the Patients with PCOS}

As illustrated in Table 2, there is a number of additional clinical features, like tiny cysts on the surface of the ovaries (polycystic) and hair symptoms ie: hirsutism due to underproduction of estrogen and overproduction of androgens (testosterone, dehydroepiandrosterone and androstenedione) by the ovaries and skin symptoms i.e.: acanthosis nigra due to insulin resistance and hyperinsulinemia [4] [11] [12] [13].

Table 2. Presenting features PCOS.

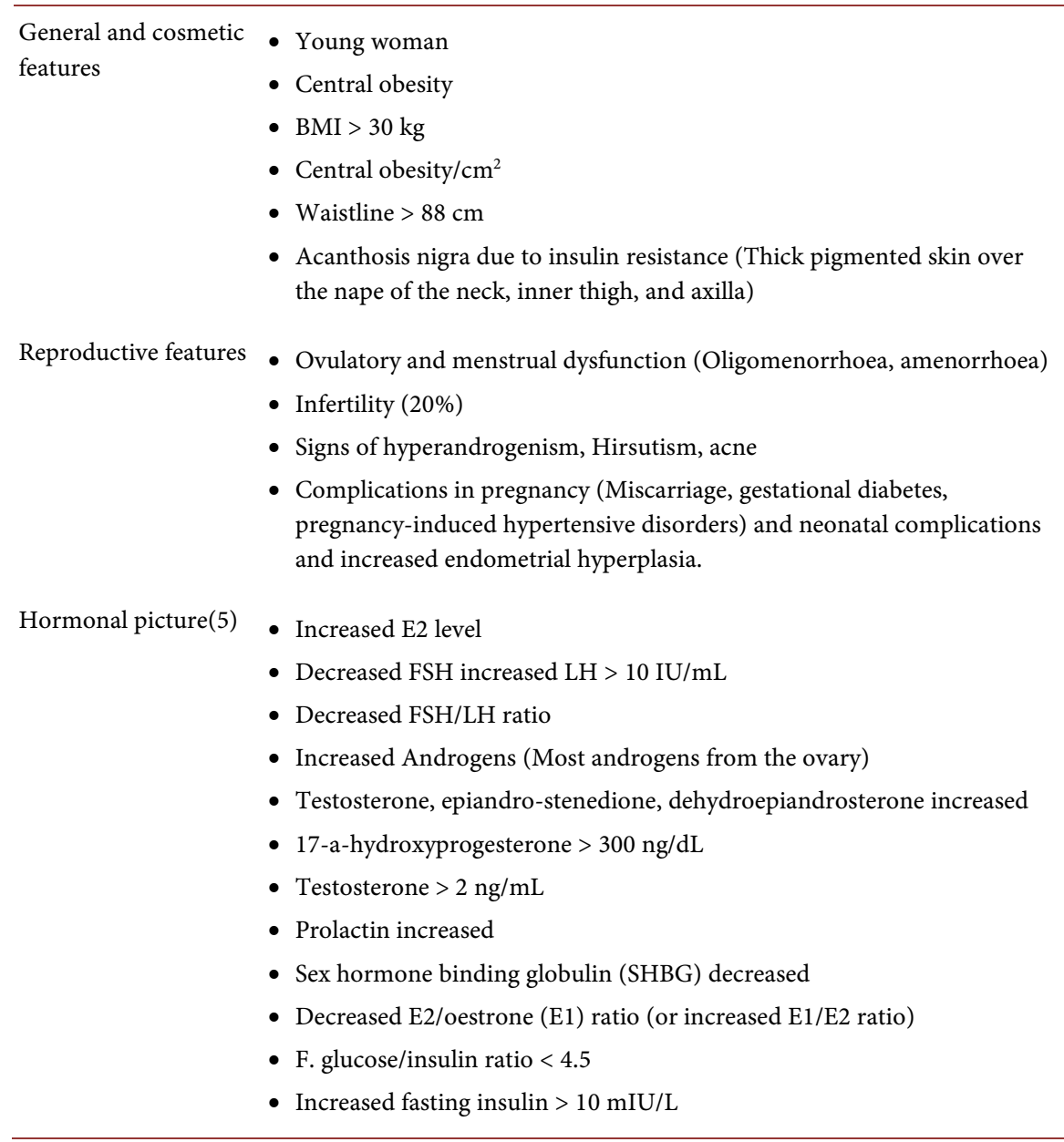




\section{Treatment to the PCOS Patients Specially Who Desires Pregnancy}

Pre-Conceptional counseling and guidelines play an initial major role in infertility treatment. The choice of the most appropriate treatment depends on the patient's age, other factors associated with infertility such as tubal patency, quality, and quantity of sperm of the husband, etc, experience and duration of previous treatments and the level of anxiety of the couple. The efficacy of the treatment of infertile women with PCOS to be optimum, tubal patency evaluation (by hysterosalpingography or laparoscopy with chromotubation) and semen analysis are mandatory to decide the suitable treatment. So treatment modalities have two measures-non-pharmacological and the pharmacological [5].

\subsection{Non-Pharmacological Measures}

\subsubsection{Assurance to the Couple}

The infertile couple remains psychologically disturbed right from the beginning more so as the investigation goes on, so the couple in such case should be tactfully handled to minimize psychological upset.

\subsubsection{Weight Reduction}

Weight reduction is considered as the fundamental treatment for infertility in obese women with PCOS [44] [45]. So lifestyle alteration in diet and exercise with optimizing the body mass index (BMI) below 25 antagonist menstrual disorders, infertility, insulin resistance, and hyperandrogenemia. A $5 \%$ to $10 \%$ loss in body weight over a period of six months regardless of BMI may be associated with improvement in central obesity, hyperandrogenism and ovulation rate [4]. Other preconceptional counseling like administering folic acid to reduce the risk of fetal neural tube defects, encouragement of physical activity and avoidance of other risk factors, such as tobacco use and alcohol consumption, should be performed.

\subsubsection{Counseling of Pregnancy-Related Complications} in Women with PCOS

As illustrated in Table 3, it has been discovered that the risk of congenital anomalies (such as heart defects and neural tube defects), gestational diabetes mellitus, hypertensive disorders during pregnancy mainly pre-eclampsia, miscarriages, preterm births, the need for intensive unit care, increased perinatal mortality and cesarean delivery is more in obese women with PCOS than the normal women [46] [47]. There is a close relationship between maternal hyperandrogenism and the risk for preterm births and preeclampsia [48].

So for the improvement of reproductive and metabolic functions, reduction in the body weight may lower the risk of pregnancy-related complications and the neonatal complications after delivery. In this context, lifestyle change should be the first choice for weight loss rather than the use of medications and bariatric surgery to reduce weight which may be associated with unwanted complications [49]. 
Table 3. Sequela of PCOS. There are numerous complications associated with PCOS and the sequelae of PCOS not limited to one system of the body.

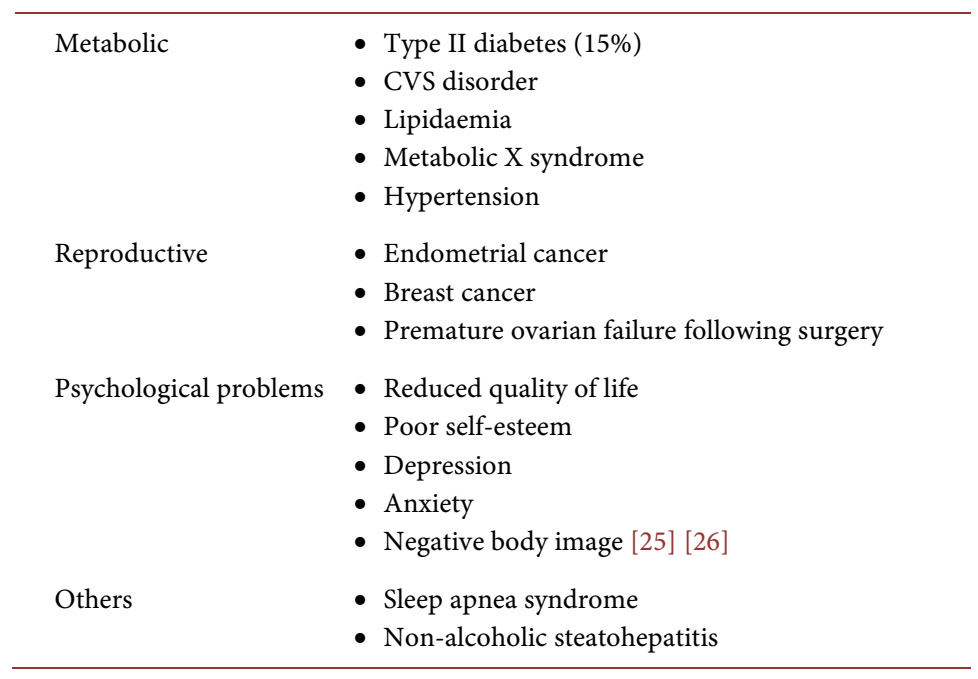

\subsection{Pharmacological Measures}

\subsubsection{Clomiphene Citrate}

Chronic anovulation is the common cause of infertility in the patient with PCOS, so induction of ovulation with clomiphene citrate remains the first-line treatment [50] [51]. This drug belongs to estrogen receptor modulator (it is anti-estrogenic as well as weakly estrogenic) and its mechanism of action as the antagonist is controversial in humans. It blocks the estrogen receptors in the hypothalamus preventing the negative feedback of endogenous estrogen which results in increased GnRH pulse amplitude causing increased endogenous gonadotropin secretion from the pituitary and the dominant follicle is recruited (follicle that has the highest number of follicle-stimulating hormone (FSH) receptors) between the sixth and ninth day of the menstrual cycle [52]. Ovaries get enlarged with the hormonal influences as result ovulation occurs. The anti-estrogenic effects of clomiphene are seen on the endometrium and on cervical mucus.

\subsubsection{Gonadotropins}

Gonadotrophin is considered as the second-line treatment for induction of ovulation. [5] The use of gonadotropins [recombinant follicle-stimulating hormone (FSHr) or human menopausal gonadotropin (HMG)] for the induction of ovulation can be followed by two methods of conception as by timed intercourse or intrauterine insemination (IUI) [50]. The prerequisites to initiate gonadotropin therapy is ovarian reserve must be present and it is always wise to evaluate tubal patency before initiating gonadotropin to stimulate ovaries if the patient has not been evaluated previously on clomiphene treatment. If the fallopian tube is found to be patent and ready for in vivo fertilization, low doses of gonadotropin can be used to prevent from the complications like ovarian hyperstimulation syndrome and multiple gestations due to higher doses [53]. Intrauterine insemination (IUI) has a higher opportunity of successful pregnancy rates than 
timed intercourse patients with the use of gonadotropins [54]. There is a little modification on treatment approach on IUI as the recombinant hCG is administered for final oocyte maturation when the dominant follicle has a mean diameter of 17 to $18 \mathrm{~mm}$ via ultra-sound examination and capacitated sperm can be injected into the uterine cavity 36 hours later which is not used in timed intercourse. But the same lower dose of gonadotropins is used as in timed intercourse. After 14 days, the level of beta hCG is checked to confirm the pregnancy.

\subsection{Laparoscopic Ovarian Drilling (LOD) Surgery}

LOD is done for cases found resistant to medical therapy and who require laparoscopy for another reason (pelvic pain, adnexal mass, etc). Ovarian surface cysts are punctured up to depth $2-4 \mathrm{~mm}$. The cysts are vaporized using monopolar cutting current. 5 - 8 punctures are made in each ovary because a larger number may favor the development of premature ovarian failure [55]. The ovarian drilling in the treatment of infertility in women with PCOS results decreased secretion of androgens and consequent reduction of peripheral aromatization of those androgens into estrone which favors the follicular growth creating estrogenic follicular microenvironment [56]. Pregnancy rates following ovarian diathermy are higher.

Clomiphene treatment should be restarted again if the patient does not regain normal ovulatory cycles after three months of ovarian drilling and the use of gonadotropins should be considered after 6 months of anovulatory cycles following the ovarian drilling procedure.

\subsection{Third-Line Treatment: In Vitro Fertilization and Embryo Transfer}

In vitro fertilization represents the third-line treatment for infertility in women with PCOS. The IVF becomes the first option in the patient with diagnosed and established bilateral tubal occlusion and/or concentration of recovered motile sperm less than or equal to 5 million. The risk of OHSS is the main complication of the high complex treatment in women with PCOS. Thus, controlled ovarian stimulation with low doses of gonadotropins is begun once the pituitary downregulation is achieved with administering of gonadotropin-releasing hormone $(\mathrm{GnRH})$ analog. Exogenous gonadotropins (uFSH, rFSH, HMG) are used for ovarian stimulation. The follicular growth response is monitored by sonographic measurement of follicles, cervical mucus study, serum estradiol estimation commencing on the $8^{\text {th }}$ day of the treatment cycle. When two or more follicles are of $17-18 \mathrm{~mm}$ in diameter and level of serum Estradiol is optimum, hCG or recombinant hCG is administered to induce oocyte maturation then the oocytes are retrieved aseptically through vaginal route under ultrasonic guidance 36 hours after the hCG is given. Approximately 50,000 - 10,000 capacitated sperms are placed into the culture media containing the oocyte within 4 - 6 hours of retrieval. The egg may demonstrate the signs of fertilization (presence of two pro-nuclei in the presence of $2^{\text {nd }}$ polar body) when examined $16-18$ hours after 
insemination. Only 2 - 3 fertilized ova (to prevent from multiple gestations) can be placed into uterine cavity close to fundus about 3 days after fertilization through a fine flexible soft catheter transcervically. Excess oocytes and embryos can be cryopreserved for future use. The luteal phase support to the embryo is maintained by progesterone supplement.

\subsection{Other Adjuvant Therapy}

Treatment with metformin (insulin sensitizer) improves the metabolic syndrome by reducing all the parameters: weight, BMI (hyperinsulinemia and hyperandrogenism), BP and lipid abnormalities. Women with PCOS and hyperinsulinemia with BMI $>25$ ovulate satisfactorily when clomiphene is combined with metformin. Although metformin is associated with better clinical pregnancy rates there is no evidence of better live birth rates when this drug is used alone or in combination with CC [57]. From a reproduction standpoint, there is also no benefit for its use in short (less than four weeks) or long (more than four weeks) periods prior to starting CC treatment in women with PCOS. Therefore, the use of metformin should be restricted to the treatment of glucose intolerance or type II diabetes in women with PCOS and should not be used to induce ovulation.

The effectiveness of aromatase inhibitors in the treatment of PCOS remains controversial. Although aromatase inhibitors have been used in women with PCOS as an alternative method to avoid the anti-estrogenic effect of CC on the endometrium, these compounds are not typically used in clinical practice to treat infertility in these patients. Their mechanism of action is based on reducing the peripheral conversion of androgens to estrogens in ovarian granulosa cells by blocking aromatase. Consequently, a decrease in estrogen serum levels and in its negative feedback in the hypothalamus and pituitary gland is noted, resulting in increased endogenous gonadotropin release [58]. Aromatase inhibitors are promising, and long-term studies are necessary to prove their safety.

\section{Conclusion}

PCOS becomes the more prevalent disorder among women of reproductive age with complications that persist lifelong. It has perplexing characteristics such as irregular or no menstrual periods, heavy periods, excess body and facial hair, acne, pelvic pain, difficulty getting pregnant, and patches of thick, darker, velvety skin etc. Infertility is the devastating complication of PCOS and it is associated with alteration of the hormones. Current treatment modalities are not able to address all aspects of PCOS. Further research is needed in genetics, pathophysiology and treatment modalities of the syndrome which could help to minimize reproductive complications especially infertility and other metabolic issues.

\section{Acknowledgements}

This work was supported by grants from the National Natural Science Foundation of China (31700736), Hubei Province Health and Family Planning Scientific 
Research Project (WJ2016Y07), Hubei Province Scientific and Technological Research Project (Q20171306), Yangtze Leading Talents Program (Xianwang Wang) and the College Students Innovative Entrepreneurial Training Program in Yangtze University (2018184).

\section{Conflicts of Interest}

The authors declare no conflicts of interest regarding the publication of this paper.

\section{References}

[1] Balen, A.H. and Rutherford, A.J. (2007) Managing Anovulatory Infertility and Polycystic Ovary Syndrome. BMJ, 335, 663-666.

https://doi.org/10.1136/bmj.39335.462303.80

[2] Balen, A. and Michelmore, K. (2002) What Is Polycystic Ovary Syndrome? Are National Views Important? Human Reproduction (Oxford, England), 17, 2219-2227. https://doi.org/10.1093/humrep/17.9.2219

[3] Rasquin Leon, L.I. and Mayrin, J.V. (2019) Polycystic Ovarian Disease (Stein-Leventhal Syndrome). StatPearls Publishing, Treasure Island.

[4] Goodarzi, M.O., Dumesic, D.A., Chazenbalk, G. and Azziz, R. (2011) Polycystic Ovary Syndrome: Etiology, Pathogenesis and Diagnosis. Nature Reviews Endocrinology, 7, 219-231. https://doi.org/10.1038/nrendo.2010.217

[5] Escobar-Morreale, H.F. (2018) Polycystic Ovary Syndrome: Definition, Aetiology, Diagnosis and Treatment. Nature Reviews Endocrinology, 14, 270-284.

https://doi.org/10.1038/nrendo.2018.24

[6] Neven, A.C.H., Laven, J., Teede, H.J. and Boyle, J.A. (2018) A Summary on Polycystic Ovary Syndrome: Diagnostic Criteria, Prevalence, Clinical Manifestations, and Management According to the Latest International Guidelines. Seminars in Reproductive Medicine, 36, 5-12. https://doi.org/10.1055/s-0038-1668085

[7] Joham, A.E., Teede, H.J., Ranasinha, S., Zoungas, S. and Boyle, J. (2015) Prevalence of Infertility and Use of Fertility Treatment in Women with Polycystic Ovary Syndrome: Data from a Large Community-Based Cohort Study. Journal of Women's Health, 24, 299-307. https://doi.org/10.1089/jwh.2014.5000

[8] Pastor, C.L., Griffin-Korf, M.L., Aloi, J.A., Evans, W.S. and Marshall, J.C. (1998) Polycystic Ovary Syndrome: Evidence for Reduced Sensitivity of the Gonadotropin-Releasing Hormone Pulse Generator to Inhibition by Estradiol and Progesterone. The Journal of Clinical Endocrinology and Metabolism, 83, 582-590. https://doi.org/10.1210/jcem.83.2.4604

[9] Velija-Asimi, Z. (2013) Evaluation of Endocrine Changes in Women with the Polycystic Ovary Syndrome during Metformin Treatment. Bosnian Journal of Basic Medical Sciences, 13, 180-185. https://doi.org/10.17305/bjbms.2013.2359

[10] Goodman, N.F., Cobin, R.H., Futterweit, W., Glueck, J.S., Legro, R.S., et al. (2015) American Association of Clinical Endocrinologists, American College of Endocrinology, and Androgen Excess and Pcos Society Disease State Clinical Review: Guide to the Best Practices in the Evaluation and Treatment of Polycystic Ovary Syndrome Part 2. Endocrine Practice: Official Journal of the American College of Endocrinology and the American Association of Clinical Endocrinologists, 21, 1415-1426. https://doi.org/10.4158/EP15748.DSCPT2 
[11] Rosenfield, R.L. and Ehrmann, D.A. (2016) The Pathogenesis of Polycystic Ovary Syndrome (PCOS): The Hypothesis of PCOS as Functional Ovarian Hyperandrogenism Revisited. Endocrine Reviews, 37, 467-520. https://doi.org/10.1210/er.2015-1104

[12] Franks, S. (2008) Polycystic Ovary Syndrome in Adolescents. International Journal of Obesity, 32, 1035-1041. https://doi.org/10.1038/ijo.2008.61

[13] Jayasena, C.N. and Franks, S. (2014) The Management of Patients with Polycystic Ovary Syndrome. Nature Reviews Endocrinology, 10, 624. https://doi.org/10.1038/nrendo.2014.102

[14] Salley, K.E., Wickham, E.P., Cheang, K.I., Essah, P.A., Karjane, N.W., et al. (2007) Glucose Intolerance in Polycystic Ovary Syndrome-A Position Statement of the Androgen Excess Society. The Journal of Clinical Endocrinology and Metabolism, 92, 4546-4556. https://doi.org/10.1210/jc.2007-1549

[15] Azziz, R., Carmina, E., Chen, Z., Dunaif, A., Laven, J.S.E., et al. (2016) Polycystic Ovary Syndrome. Nature Reviews Disease Primers, 2, 16057. https://doi.org/10.1038/nrdp.2016.57

[16] Bednarska, S. and Siejka, A. (2017) The Pathogenesis and Treatment of Polycystic Ovary Syndrome: What's New? Advances in Clinical and Experimental Medicine: Official Organ Wroclaw Medical University, 26, 359-367. https://doi.org/10.17219/acem/59380

[17] Cooney, L.G. and Dokras, A. (2017) Depression and Anxiety in Polycystic Ovary Syndrome: Etiology and Treatment. Current Psychiatry Reports, 19, 83. https://doi.org/10.1007/s11920-017-0834-2

[18] Wright, C.E., Zborowski, J.V., Talbott, E.O., McHugh-Pemu, K. and Youk, A. (2004) Dietary Intake, Physical Activity, and Obesity in Women with Polycystic Ovary Syndrome. International Journal of Obesity, 28, 1026. https://doi.org/10.1038/sj.ijo.0802661

[19] Zhang, L., Ming, L. and Yu, J. (2007) BH3 Mimetics to Improve Cancer Therapy; Mechanisms and Examples. Drug Resistance Updates: Reviews and Commentaries in Antimicrobial and Anticancer Chemotherapy, 10, 207-217. https://doi.org/10.1016/j.drup.2007.08.002

[20] Zhao, S., Tian, Y., Gao, X., Zhang, X., Liu, H., et al. (2015) Family-Based Analysis of Eight Susceptibility Loci in Polycystic Ovary Syndrome. Scientific Reports, 5, Article No. 12619. https://doi.org/10.1038/srep12619

[21] Sirmans, S.M. and Pate, K.A. (2013) Epidemiology, Diagnosis, and Management of Polycystic Ovary Syndrome. Clinical Epidemiology, 6, 1-13. https://doi.org/10.2147/CLEP.S37559

[22] Ercan, C.M., Coksuer, H., Aydogan, U., Alanbay, I., Keskin, U., et al. (2013) Sexual Dysfunction Assessment and Hormonal Correlations in Patients with Polycystic Ovary Syndrome. International Journal of Impotence Research, 25, 127.

https://doi.org/10.1038/ijir.2013.2

[23] Dunaif, A. and Fauser, B.C. (2013) Renaming PCOS-A Two-State Solution. The Journal of Clinical Endocrinology and Metabolism, 98, 4325-4328. https://doi.org/10.1210/jc.2013-2040

[24] Gaasenbeek, M., Powell, B.L., Sovio, U., Haddad, L., Gharani, N., et al. (2004) Large-Scale Analysis of the Relationship between CYP11A Promoter Variation, Polycystic Ovarian Syndrome, and Serum Testosterone. The Journal of Clinical Endocrinology and Metabolism, 89, 2408-2413. https://doi.org/10.1210/jc.2003-031640 
[25] Urbanek, M. (2007) The Genetics of the Polycystic Ovary Syndrome. Nature Clinical Practice Endocrinology \& Metabolism, 3, 103.

https://doi.org/10.1038/ncpendmet0400

[26] Filippou, P. and Homburg, R. (2017) Is Foetal Hyperexposure to Androgens a Cause of PCOS? Human Reproduction Update, 23, 421-432.

https://doi.org/10.1093/humupd/dmx013

[27] Dokshin, G.A., Baltus, A.E., Eppig, J.J. and Page, D.C. (2013) Oocyte Differentiation Is Genetically Dissociable from Meiosis in Mice. Nature Genetics, 45, 877. https://doi.org/10.1038/ng.2672

[28] Nagaoka, S.I., Hassold, T.J. and Hunt, P.A. (2012) Human Aneuploidy: Mechanisms and New Insights into an Age-Old Problem. Nature Reviews Genetics, 13, 493. https://doi.org/10.1038/nrg3245

[29] Fortune, J.E., Cushman, R.A., Wahl, C.M. and Kito, S. (2000) The Primordial to Primary Follicle Transition. Molecular and Cellular Endocrinology, 163, 53-60. https://doi.org/10.1016/S0303-7207(99)00240-3

[30] Peters, H., Byskov, A.G., Himelstein-Braw, R. and Faber, M. (1975) Follicular Growth: The Basic Event in the Mouse and Human Ovary. Journal of Reproduction and Fertility, 45, 559-566. https://doi.org/10.1530/jrf.0.0450559

[31] Magoffin, D.A. (2005) Ovarian Theca Cell. The International Journal of Biochemistry \& Cell Biology, 37, 1344-1349. https://doi.org/10.1016/j.biocel.2005.01.016

[32] Edson, M.A., Nagaraja, A.K. and Matzuk, M.M. (2009) The Mammalian Ovary from Genesis to Revelation. Endocrine Reviews, 30, 624-712.

https://doi.org/10.1210/er.2009-0012

[33] Barbieri, R.L., Makris, A., Randall, R.W., Daniels, G., Kistner, R.W., et al. (1986) Insulin Stimulates Androgen Accumulation in Incubations of Ovarian Stroma Obtained from Women with Hyperandrogenism. The Journal of Clinical Endocrinology and Metabolism, 62, 904-910. https://doi.org/10.1210/jcem-62-5-904

[34] Willis, D.S., Watson, H., Mason, H.D., Galea, R., Brincat, M., et al. (1998) Premature Response to Luteinizing Hormone of Granulosa Cells from Anovulatory Women with Polycystic Ovary Syndrome: Relevance to Mechanism of Anovulation. The Journal of Clinical Endocrinology and Metabolism, 83, 3984-3991.

https://doi.org/10.1210/jcem.83.11.5232

[35] Ehrmann, D.A., Liljenquist, D.R., Kasza, K., Azziz, R., Legro, R.S., et al. (2006) Prevalence and Predictors of the Metabolic Syndrome in Women with Polycystic Ovary Syndrome. The Journal of Clinical Endocrinology and Metabolism, 91, 48-53. https://doi.org/10.1210/jc.2005-1329

[36] Azziz, R., Carmina, E., Dewailly, D., Diamanti-Kandarakis, E., Escobar-Morreale, H.F., et al. (2006) Positions Statement: Criteria for Defining Polycystic Ovary Syndrome as a Predominantly Hyperandrogenic Syndrome: An Androgen Excess Society Guideline. The Journal of Clinical Endocrinology and Metabolism, 91, 4237-4245. https://doi.org/10.1210/jc.2006-0178

[37] Norman, R.J., Dewailly, D., Legro, R.S. and Hickey, T.E. (2007) Polycystic Ovary Syndrome. The Lancet (London, England), 370, 685-697. https://doi.org/10.1016/S0140-6736(07)61345-2

[38] Cook, H., Brennan, K. and Azziz, R. (2011) Reanalyzing the Modified Ferriman-Gallwey Score: Is There a Simpler Method for Assessing the Extent of Hirsutism? Fertility and Sterility, 96, 1266-1270. https://doi.org/10.1016/j.fertnstert.2011.08.022 
[39] Brassard, M., AinMelk, Y. and Baillargeon, J.P. (2008) Basic Infertility Including Polycystic Ovary Syndrome. The Medical Clinics of North America, 92, 1163-1192. https://doi.org/10.1016/j.mcna.2008.04.008

[40] Cedergren, M.I. (2007) Optimal Gestational Weight Gain for Body Mass Index Categories. Obstetrics and Gynecology, 110, 759-764. https://doi.org/10.1097/01.AOG.0000279450.85198.b2

[41] Wang, R. and Mol, B.W. (2017) The Rotterdam Criteria for Polycystic Ovary Syndrome: Evidence-Based Criteria? Human Reproduction (Oxford, England), 32, 261-264. https://doi.org/10.1093/humrep/dew287

[42] Dewailly, D. (2016) Diagnostic Criteria for PCOS: Is There a Need for a Rethink? Best Practice \& Research Clinical Obstetrics \& Gynaecology, 37, 5-11. https://doi.org/10.1016/j.bpobgyn.2016.03.009

[43] Bachanek, M., Abdalla, N., Cendrowski, K. and Sawicki, W. (2015) Value of Ultrasonography in the Diagnosis of Polycystic Ovary Syndrome-Literature Review. Journal of Ultrasonography, 15, 410-422. https://doi.org/10.15557/JoU.2015.0038

[44] Moran, L.J., Pasquali, R., Teede, H.J., Hoeger, K.M. and Norman, R.J. (2009) Treatment of Obesity in Polycystic Ovary Syndrome: A Position Statement of the Androgen Excess and Polycystic Ovary Syndrome Society. Fertility and Sterility, 92, 1966-1982. https://doi.org/10.1016/j.fertnstert.2008.09.018

[45] Kumar, P. and Arora, S. (2014) Orlistat in Polycystic Ovarian Syndrome Reduces Weight with Improvement in Lipid Profile and Pregnancy Rates. Journal of Human Reproductive Sciences, 7, 255-261. https://doi.org/10.4103/0974-1208.147492

[46] Boomsma, C.M., Eijkemans, M.J., Hughes, E.G., Visser, G.H., Fauser, B.C., et al. (2006) A Meta-Analysis of Pregnancy Outcomes in Women with Polycystic Ovary Syndrome. Human Reproduction Update, 12, 673-683. https://doi.org/10.1093/humupd/dml036

[47] Qin, J.Z., Pang, L.H., Li, M.J., Fan, X.J., Huang, R.D., et al. (2013) Obstetric Complications in Women with Polycystic Ovary Syndrome: A Systematic Review and Meta-Analysis. Reproductive Biology and Endocrinology, 11, 56. https://doi.org/10.1186/1477-7827-11-56

[48] Naver, K.V., Grinsted, J., Larsen, S.O., Hedley, P.L., Jorgensen, F.S., et al. (2014) Increased Risk of Preterm Delivery and Pre-Eclampsia in Women with Polycystic Ovary Syndrome and Hyperandrogenaemia. BJOG: An International Journal of Obstetrics and Gynaecology, 121, 575-581. https://doi.org/10.1111/1471-0528.12558

[49] Roos, N., Neovius, M., Cnattingius, S., Trolle Lagerros, Y., Saaf, M., et al. (2013) Perinatal Outcomes after Bariatric Surgery: Nationwide Population Based Matched Cohort Study. BMJ, 347, f6460. https://doi.org/10.1136/bmj.f6460

[50] (2008) Consensus on Infertility Treatment Related to Polycystic Ovary Syndrome. Fertility and Sterility, 89, 505-522. https://doi.org/10.1016/j.fertnstert.2007.09.041

[51] Perales-Puchalt, A. and Legro, R.S. (2013) Ovulation Induction in Women with Polycystic Ovary Syndrome. Steroids, 78, 767-772. https://doi.org/10.1016/j.steroids.2013.05.005

[52] Imani, B., Eijkemans, M.J., te Velde, E.R., Habbema, J.D. and Fauser, B.C. (2002) A Nomogram to Predict the Probability of Live Birth after Clomiphene Citrate Induction of Ovulation in Normogonadotropic Oligoamenorrheic Infertility. Fertility and Sterility, 77, 91-97. https://doi.org/10.1016/S0015-0282(01)02929-6

[53] Balen, A.H. (2013) Ovulation Induction in the Management of Anovulatory Polycystic Ovary Syndrome. Molecular and Cellular Endocrinology, 373, 77-82. https://doi.org/10.1016/j.mce.2012.10.008 
[54] Veltman-Verhulst, S.M., Cohlen, B.J., Hughes, E. and Heineman, M.J. (2012) Intra-Uterine Insemination for Unexplained Subfertility. The Cochrane Database of Systematic Reviews, No. 2, Cd001838. https://doi.org/10.1002/14651858.CD001838.pub4

[55] Farquhar, C., Brown, J. and Marjoribanks, J. (2012) Laparoscopic Drilling by Diathermy or Laser for Ovulation Induction in Anovulatory Polycystic Ovary Syndrome. The Cochrane Database of Systematic Reviews, No. 6, Cd001122. https://doi.org/10.1002/14651858.CD001122.pub4

[56] Aakvaag, A. and Gjonnaess, H. (1985) Hormonal Response to Electrocautery of the Ovary in Patients with Polycystic Ovarian Disease. British Journal of Obstetrics and Gynaecology, 92, 1258-1264. https://doi.org/10.1111/j.1471-0528.1985.tb04872.x

[57] Tang, T., Lord, J.M., Norman, R.J., Yasmin, E. and Balen, A.H. (2012) Insulin-Sensitising Drugs (Metformin, Rosiglitazone, Pioglitazone, D-chiro-inositol) for Women with Polycystic Ovary Syndrome, Oligo Amenorrhoea and Subfertility. The Cochrane Database of Systematic Reviews, 11, Cd003053. https://doi.org/10.1002/14651858.CD003053.pub5

[58] Misso, M.L., Wong, J.L., Teede, H.J., Hart, R., Rombauts, L., et al. (2012) Aromatase Inhibitors for PCOS: A Systematic Review and Meta-Analysis. Human Reproduction Update, 18, 301-312. https://doi.org/10.1093/humupd/dms003 